\title{
A MULTI-CORE FPGA-BASED 2D-CLUSTERING ALGORITHM FOR HIGH-THROUGHPUT DATA-INTENSIVE APPLICATIONS
}

\section{Calliope-Louisa Sotiropoulou}

Aristotle University of Thessaloniki

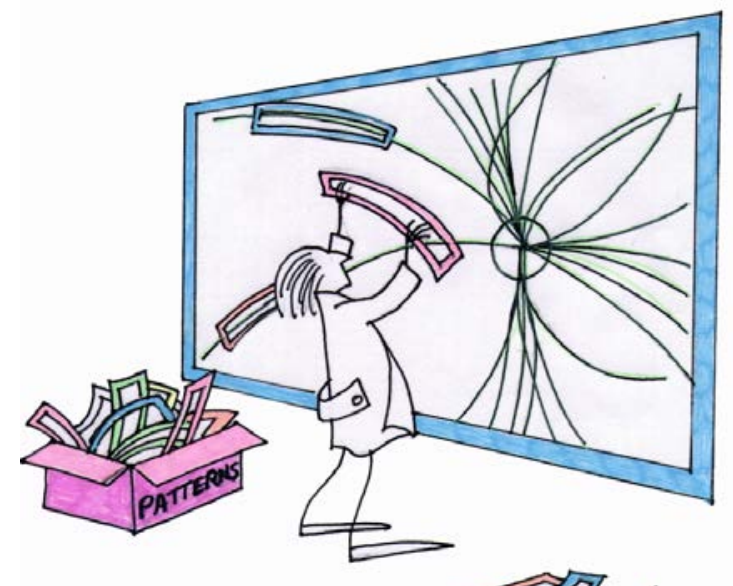




\section{Clustering Modules - FTK IM Board}

- Introduction - Motive

- ATLAS Application - FTK IM Board

- Clustering Problem

- Clustering Modules

- Parallelization 


\section{Introduction to 2D-Clustering}

- High Resolution Pixel Detectors are used for a variety of applications:

- High Energy Physics (Particle Tracking)

- Medical Imaging and Biomedical Applications

- Astrophysics Image Acquisition etc.

- 2D-Clustering is beneficial for all the above applications:

- A preprocessing step for data reduction ( $\mathrm{N}$ hits $\rightarrow 1$ cluster)

- Image segmentation process

- Increasing resolution (e.g. spatial or other parameters)

- Identifying patterns (e.g. object identification)

- Performing cluster analysis 


\section{D-Clustering for the ATLAS FastTracKer Processor}

- Main challenge: input rate $308 \mathrm{Gbits}$

- 256 S-link fibers from all pixel RODs

- Running at 1.2 Gbits (total 308Gbi

- 32bit words at $40 \mathrm{MHz}, 1$ hit a

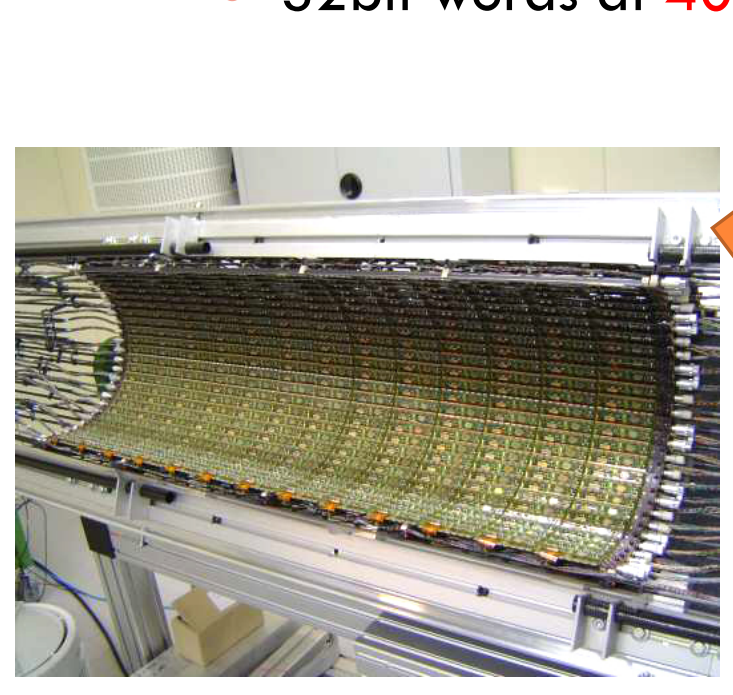

ATLAS Pixel Layer 2

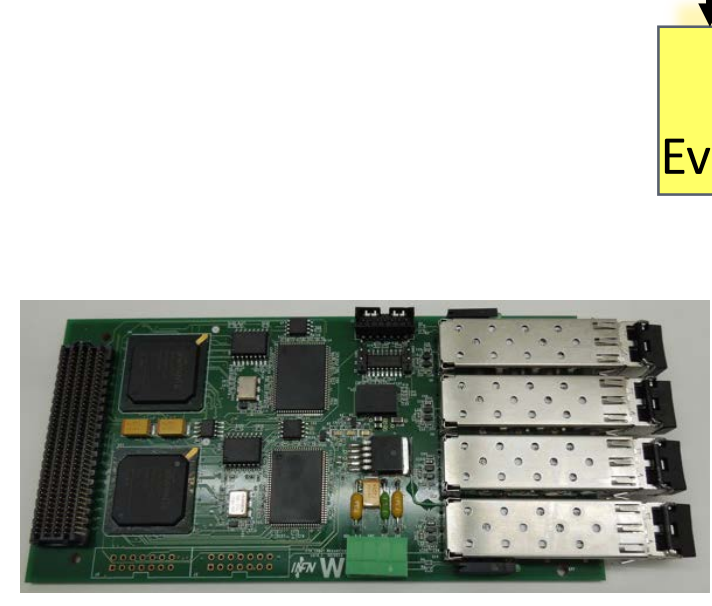

\section{PixelDetector}

WWW

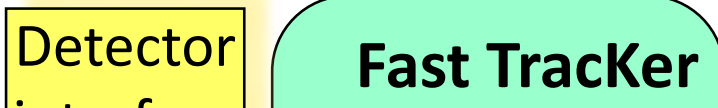
interface IIII IIIII $50 \sim 100 \mathrm{kHz}$ event rate

|||||

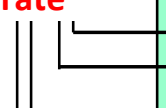
input stage

FTK Input Mezzanine Board 


\section{D-Clustering for the ATLAS FastTracKer Processor}

- Main challenge: input rate $308 \mathrm{Gbits}$

- 256 S-link fibers from all pixel RODs

- Running at 1.2 Gbits (total 308Gbi

- 32bit words at $40 \mathrm{MHz}, 1$ hit a

\section{PixelDetector}

WWW

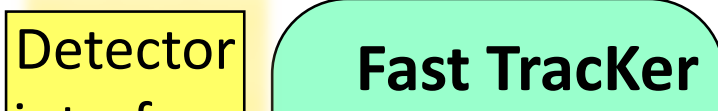
interface IIII IIII $50 \sim 100 \mathrm{kHz}$ event rate

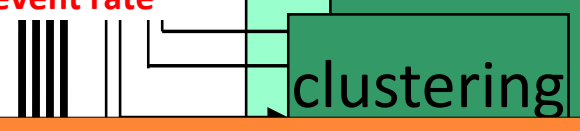

\section{Target:}

- A system which clusters data on the fly

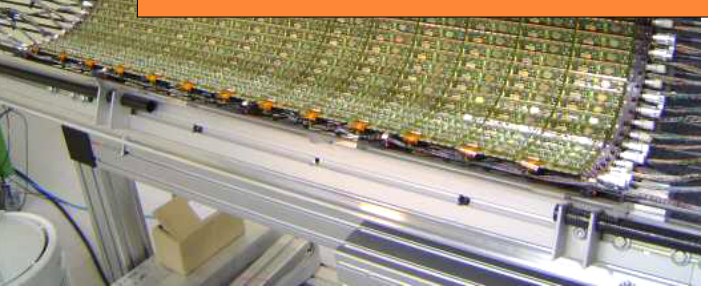

ATLAS Pixel Layer 2

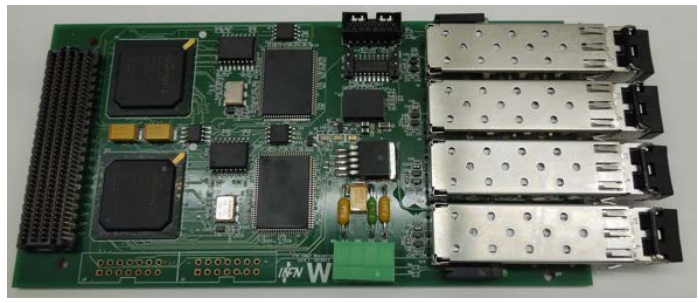

FTK Input Mezzanine Board
Level-2

Event buff 


\section{The ATLAS Pixel Module}

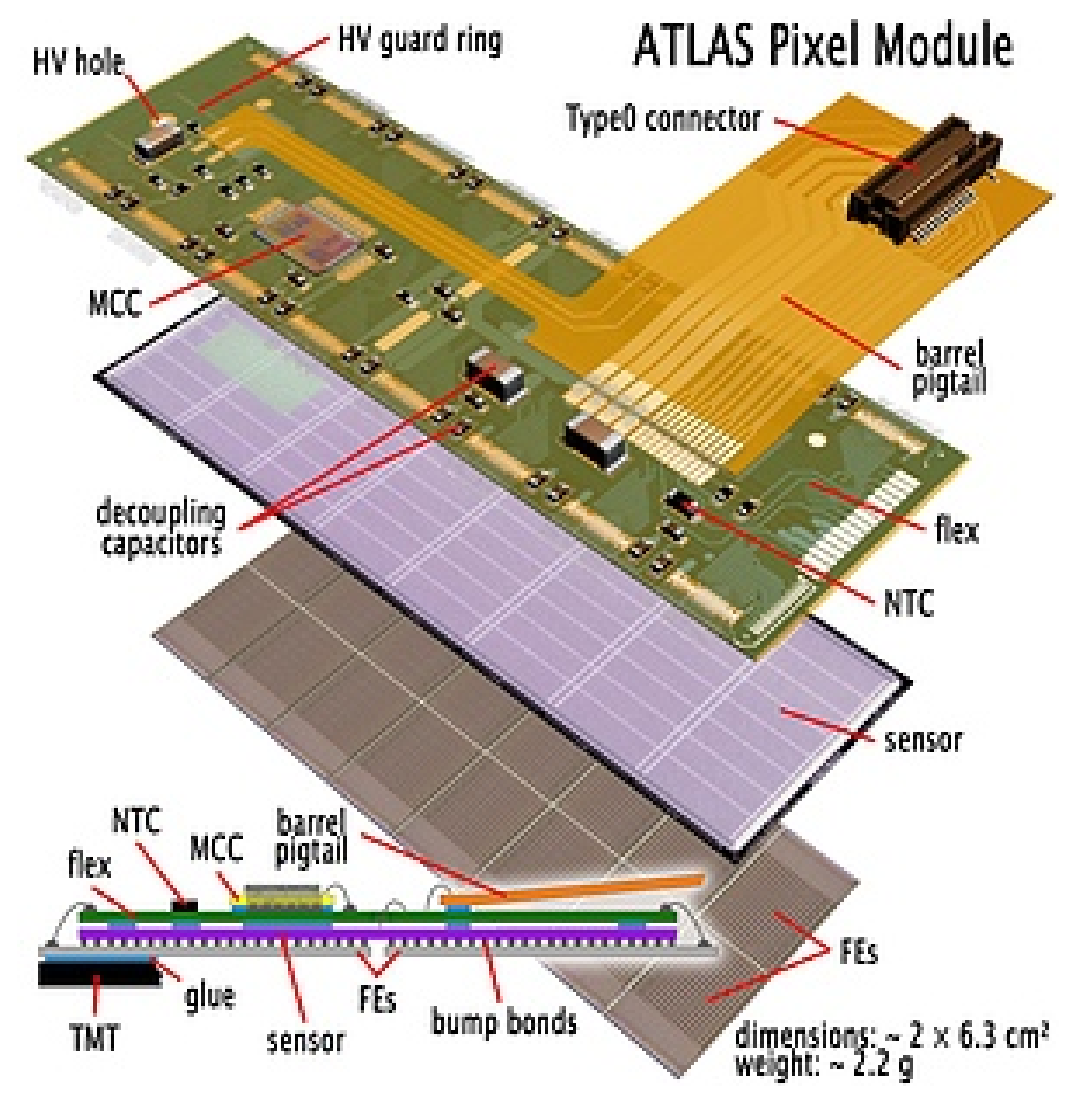

- 328x144 pixels readout by 16 Front End (FE) chips

- Each module is comprised of 2 rows of $8 \mathrm{FE}$

- Each FE reads out pixel hits by double column

- The hits in the double column are scrambled

- The pixels are 50um x 400um

- Pixels with larger occupancy (column 0 and 17 of each FE chip) are 600um wide 


\section{The ATLAS Pixel Modules}

- Expected cluster sizes on the Pixel module from single particle simulation

2D Occupancy Plot

$\eta$-Region

\begin{tabular}{|c|c|c|c|c|c|}
\hline & $0.0-0.5$ & $0.5-1.0$ & $1.0-1.5$ & $1.5-2.0$ & $2.0-2.5$ \\
\hline$\square$ & 51.9 & 32.8 & 6.7 & 0.4 & 0.5 \\
\hline$\square$ & 31.1 & 21.3 & 4.0 & 0.4 & 0.3 \\
\hline$E$ & 2.0 & 0.9 & 0.4 & 0.6 & 0.6 \\
\hline$\square$ & 9.2 & 27.3 & 48.0 & 20.8 & 0.3 \\
\hline \begin{tabular}{l|l} 
& \\
\end{tabular} & 4.8 & 16.9 & 34.7 & 15.2 & 0.4 \\
\hline \begin{tabular}{|l|l|} 
& \\
& \\
\end{tabular} & 0.7 & 0.6 & 0.7 & 0.7 & 0.9 \\
\hline \begin{tabular}{l|l|l}
$\square$ & \\
\end{tabular} & 0.0 & 0.0 & 3.0 & 34.2 & 16.7 \\
\hline \begin{tabular}{|l|l|l|} 
& & \\
\end{tabular} & 0.1 & 0.1 & 2.0 & 25.4 & 14.0 \\
\hline \begin{tabular}{|l|l|l|} 
& & \\
& & \\
\end{tabular} & 0.1 & 0.1 & 0.3 & 0.6 & 1.0 \\
\hline \begin{tabular}{l|l|l|l|}
$\square$ & 1 & 1
\end{tabular} & 0.0 & 0.0 & 0.0 & 0.7 & 34.1 \\
\hline \begin{tabular}{|l|l|l|l|} 
& & & \\
\end{tabular} & 0.0 & 0.0 & 0.0 & 0.7 & 30.4 \\
\hline \begin{tabular}{|l|l|l|l|} 
& & & \\
\end{tabular} & 0.1 & 0.0 & 0.2 & 0.3 & 0.8 \\
\hline
\end{tabular}

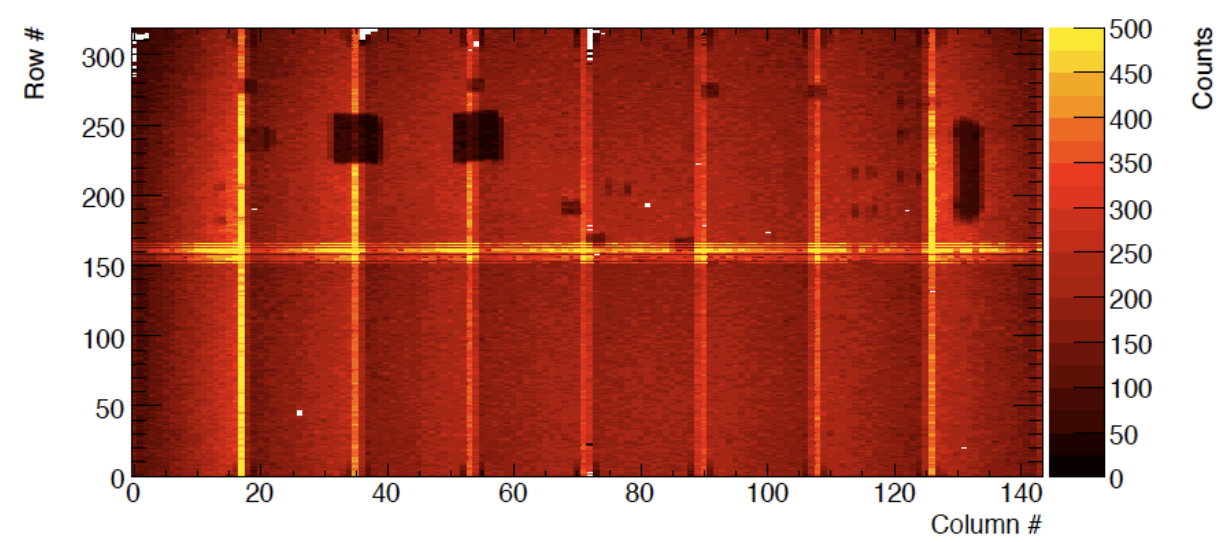

- Small probability of large clusters with a size 10-30 pixels wide (Especially in the r-phi direction) 


\section{The Clustering Problem}

\begin{tabular}{|l|l|l|l|l|l|l|l|l|l|}
\hline & 3 & 9 & 7 & & & & & & \\
\hline & 1 & & & & & 13 & 15 & & \\
\hline & & & & & & & & & \\
\hline & 4 & 8 & 6 & 11 & & & & & \\
\hline & & & & & & & 12 & & \\
\hline 2 & 5 & 10 & & & & & 14 & & \\
\hline
\end{tabular}

The Cluster Hits arrive scrambled $\rightarrow$ Loop over the list of hits
1. Associate hits from same cluster

- Loop over hit list

- Time increases with occupancy \& instantaneous luminosity

- Non linear execution time

2. Calculate cluster properties

- e.g. center, size, shape ...

- Goal:

Keep up with $40 \mathrm{Mhz}$ hit input rate

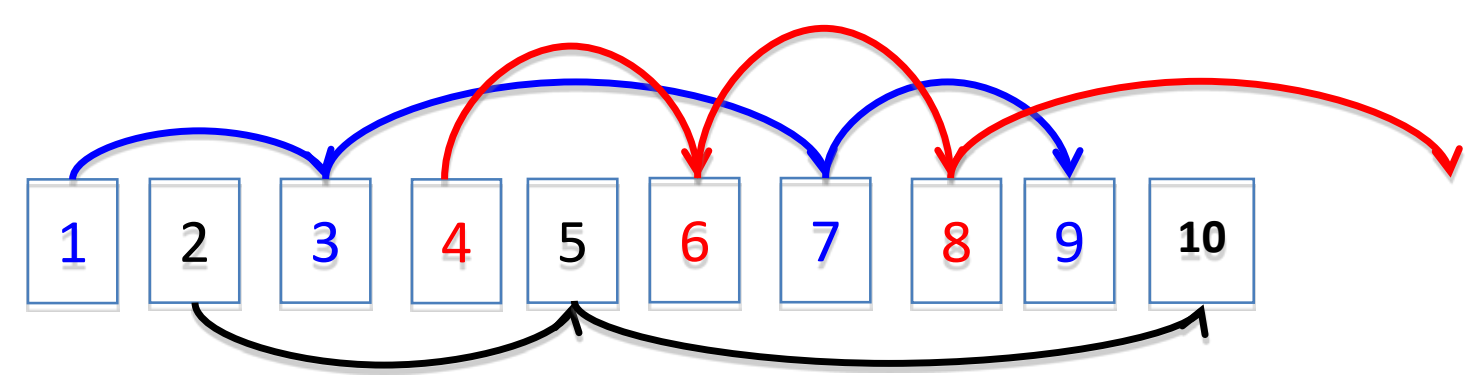




\section{Clustering Modules}
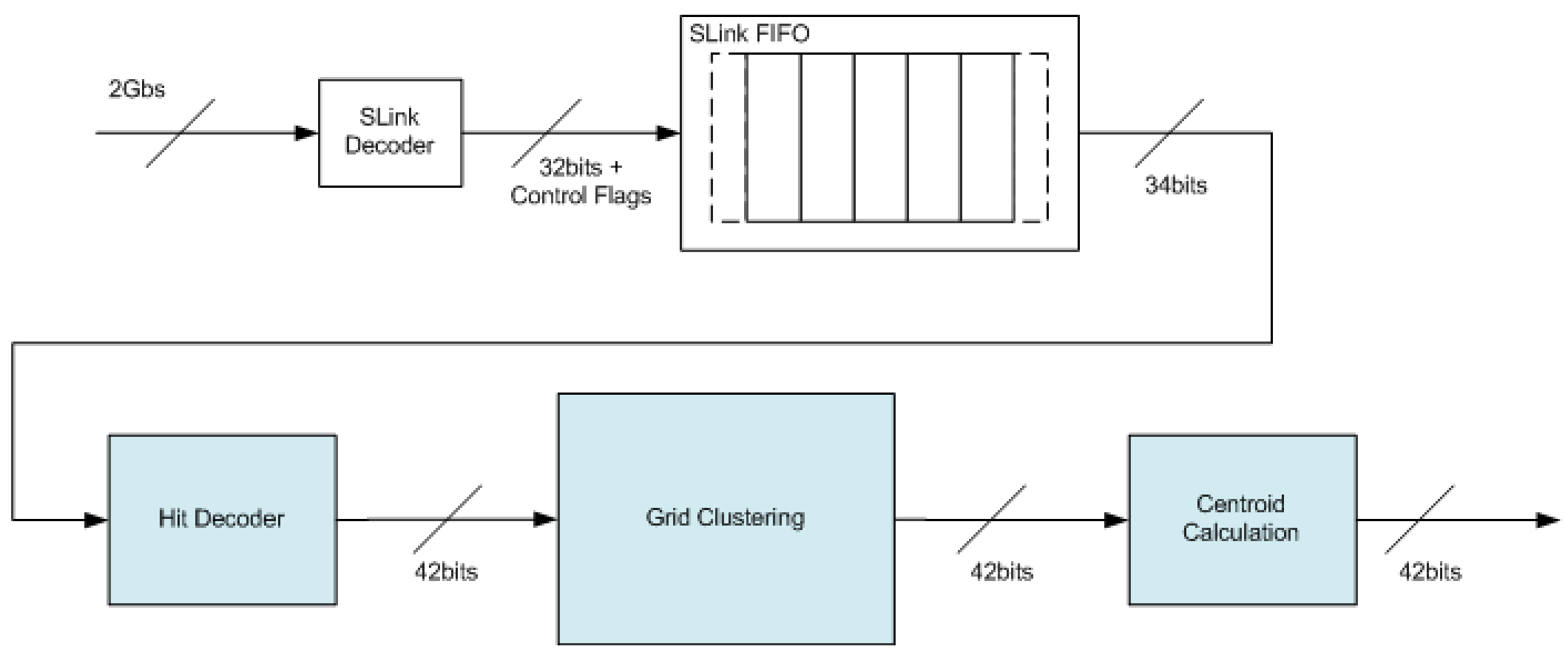


\section{Clustering Modules}
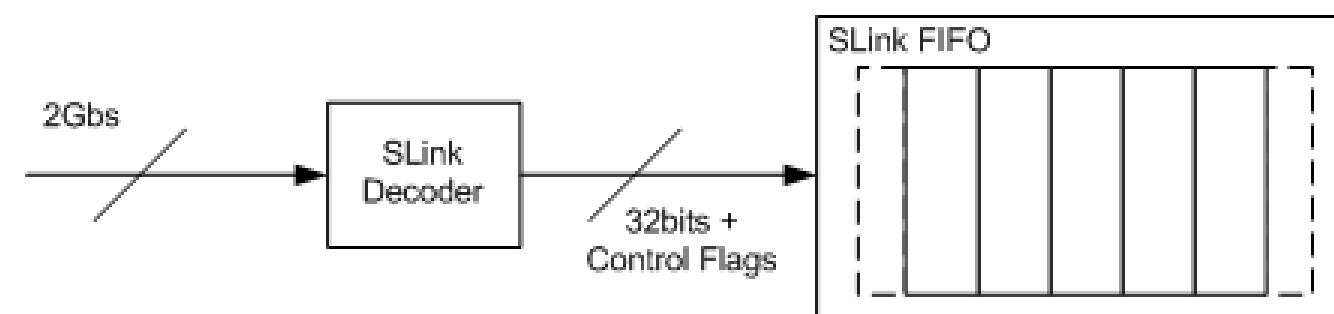

34 bits

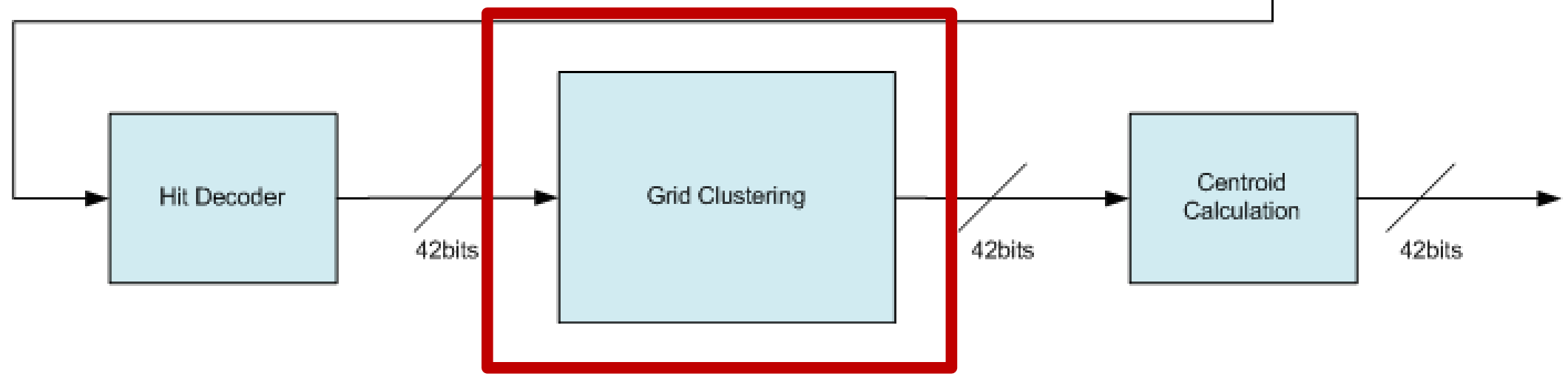




\section{Grid Clustering - Generate Window}

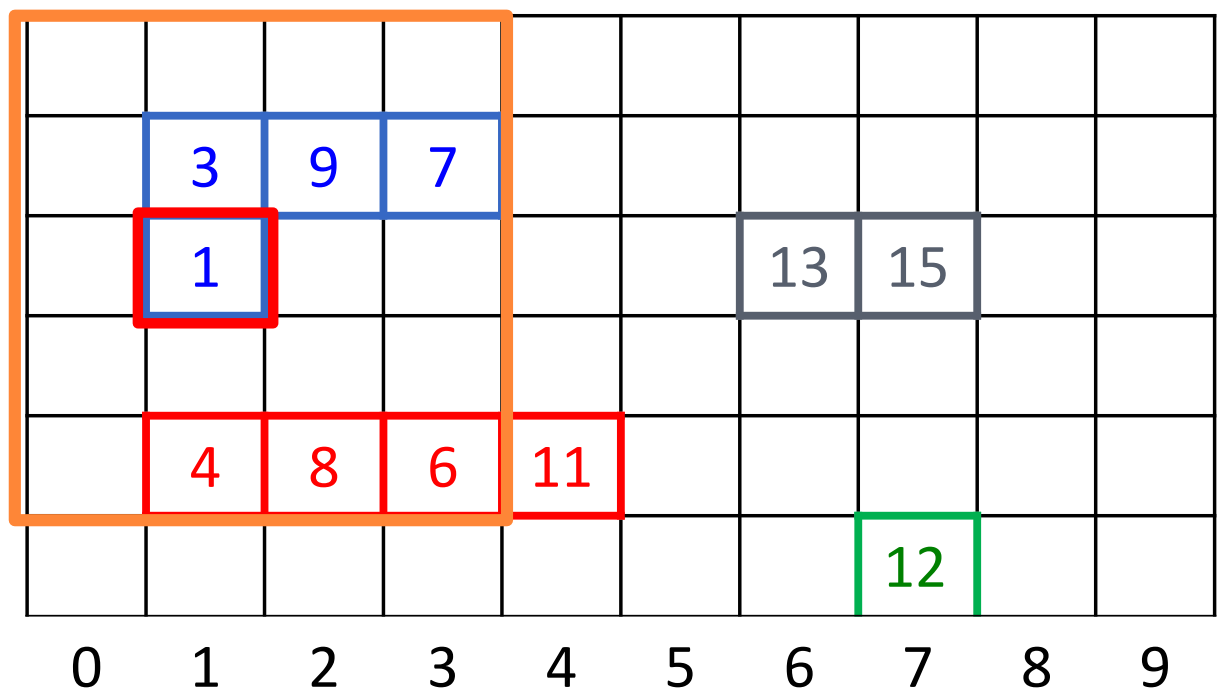

- Generate a cluster window (e.g. $4 \times 5$ pixels) around a reference hit

- The reference hit is located on the middle row of the window and

- Column 1 of the window if it belongs to an odd column 


\section{Grid Clustering - Generate Window}

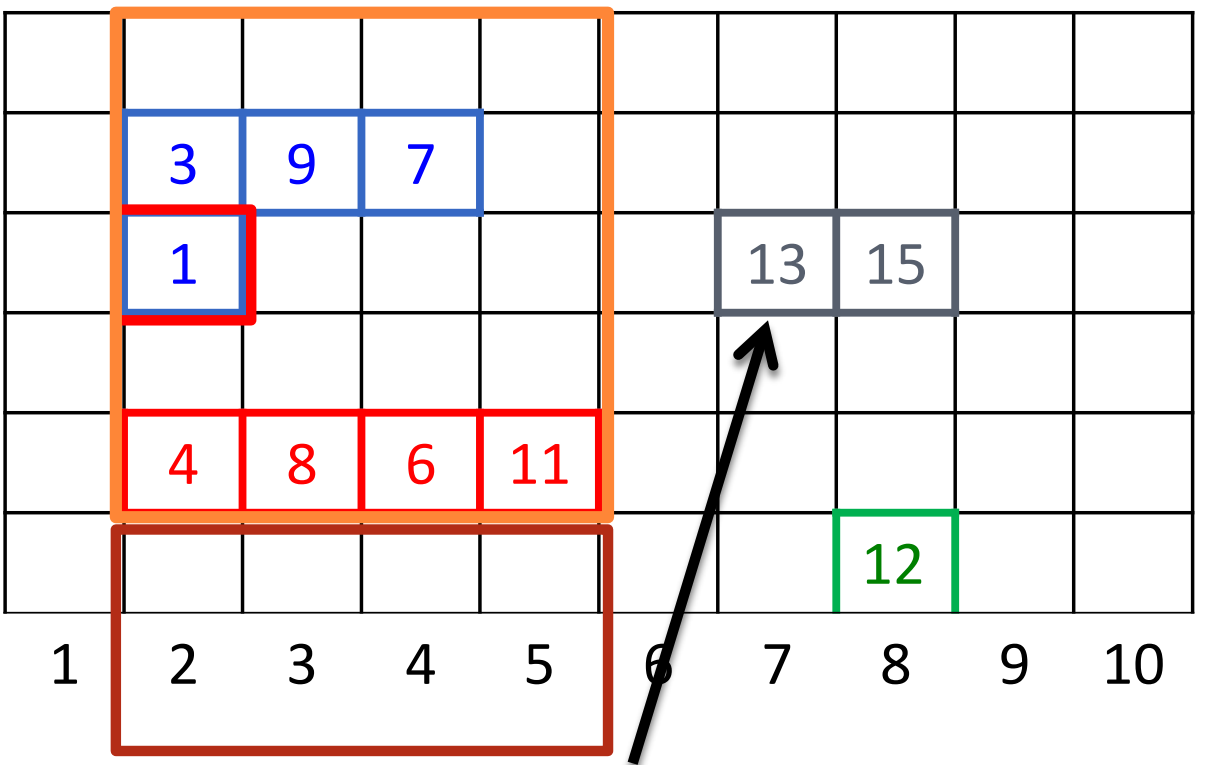

- Hits are read from the input until a hit which belongs to a column beyond the cluster window is identified

- The hits that belong to the same columns as the cluster window are stored in a separate circular buffer
- Generate a cluster window (e.g. $4 \times 5$ pixels) around a reference hit

- The reference hit is located on the middle row of the window and

- Column 1 of the window if it belongs to an odd column

- Column $\mathbf{0}$ of the window if it belongs to an even column 


\section{Grid Clustering - Select and Readout}

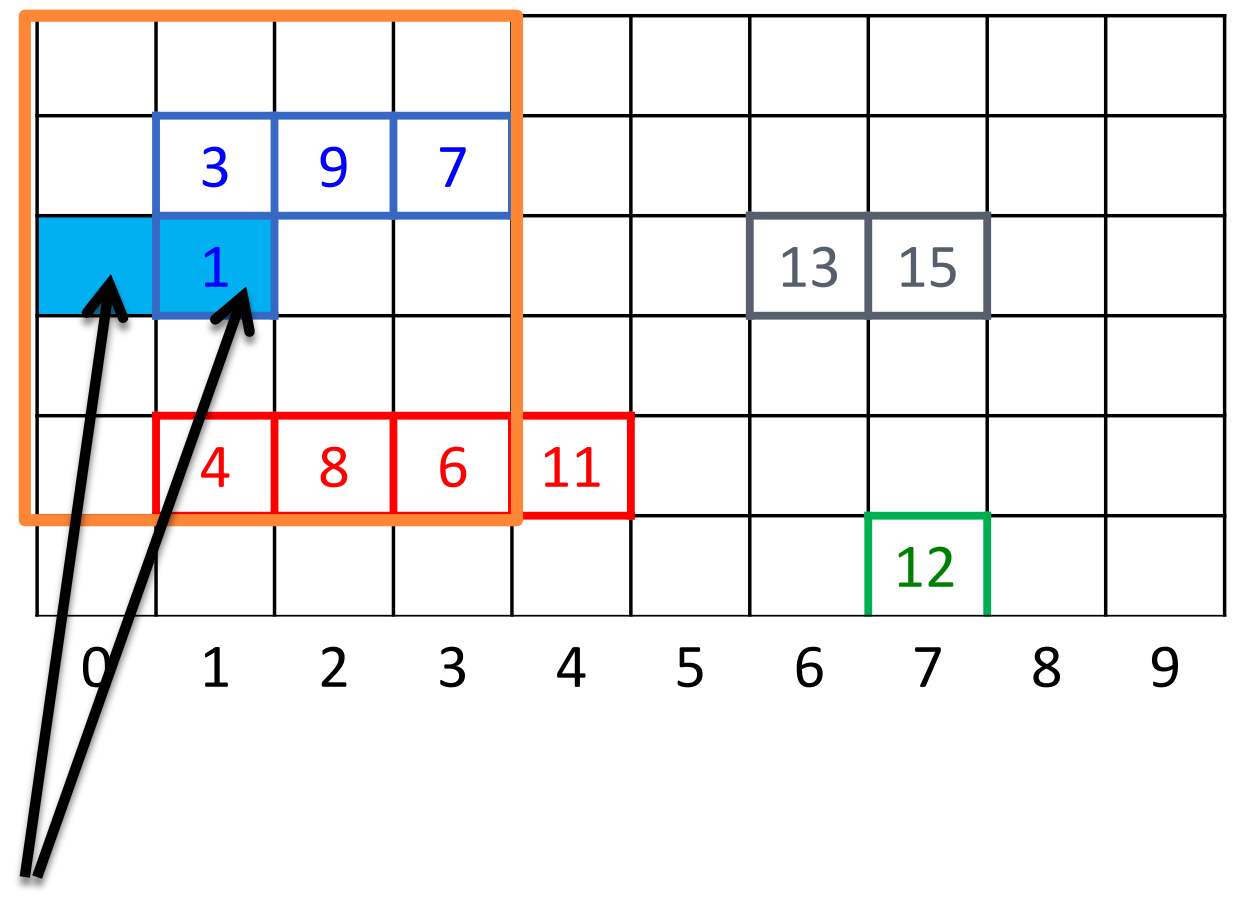

- Two cells in the cluster window are selected as "seeds" $(0$, mid-row $)$ and $(1$, mid-row)

Selected grid cells 


\section{Grid Clustering - Select and Readout}

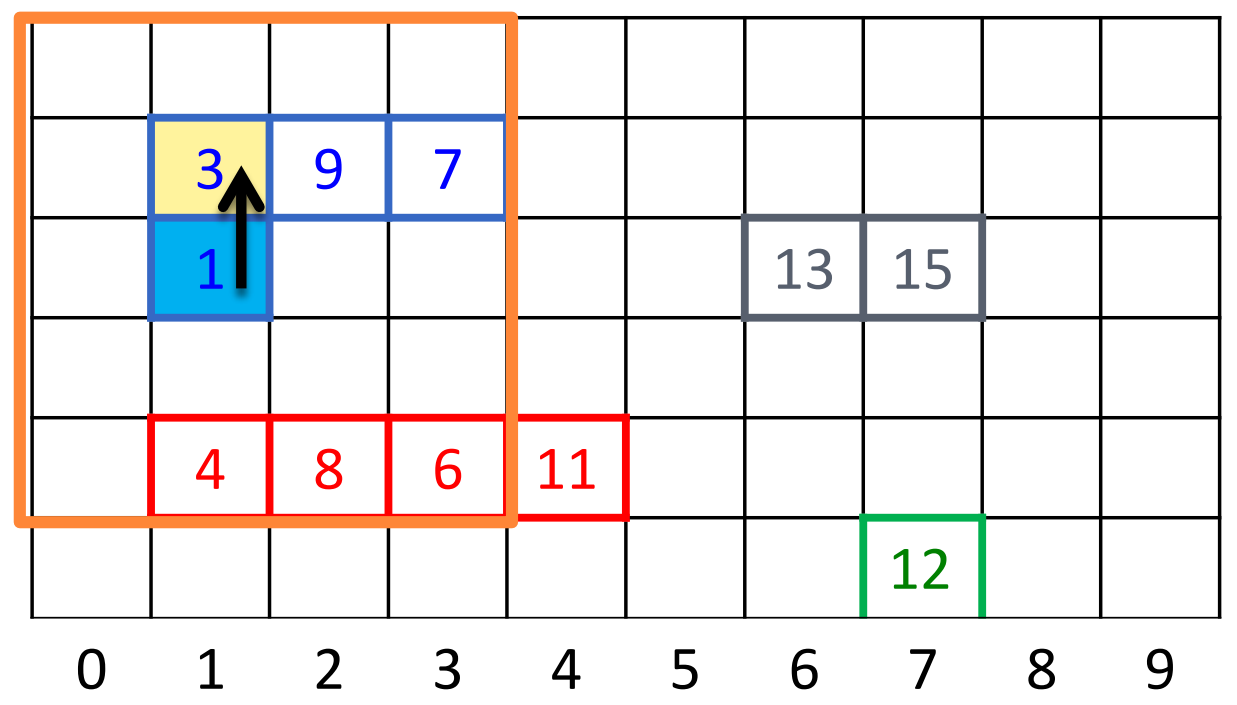

- Two cells in the cluster window are selected as "seeds" $(0$, mid-row) and (1, mid-row)

- The "selected" state is propagated to the neighboring hits 


\section{Grid Clustering - Select and Readout}

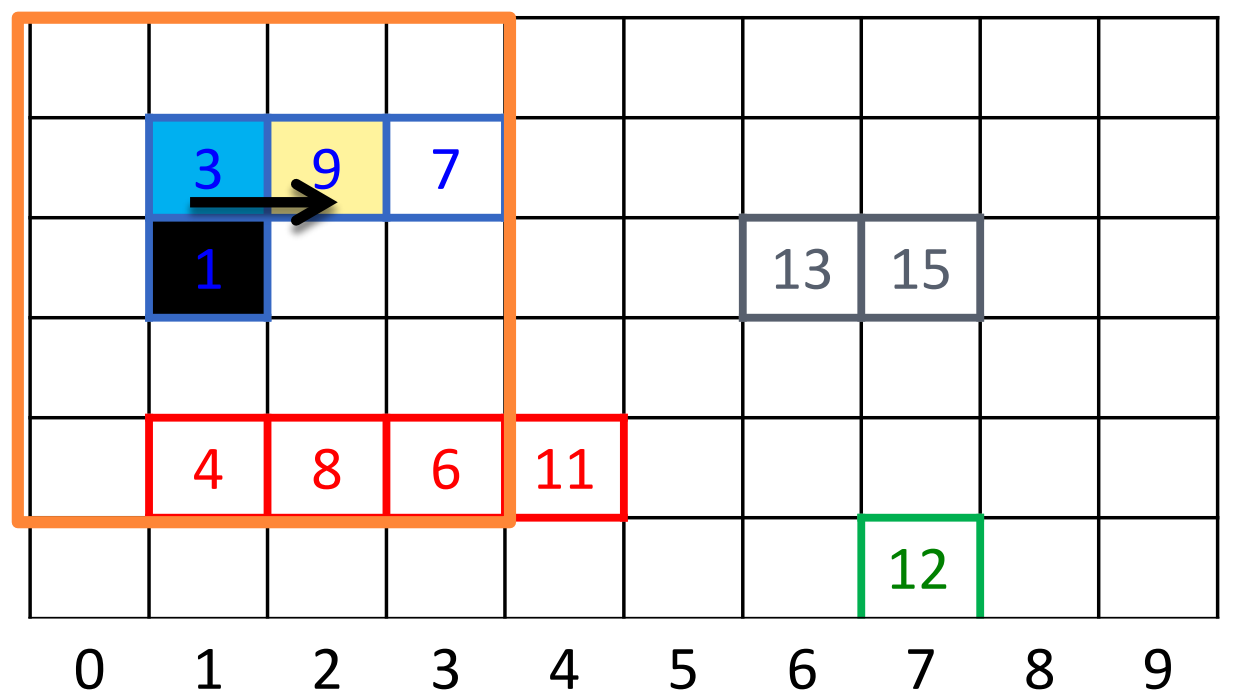

- Two cells in the cluster window are selected as "seeds" $(0$, mid-row) and (1, mid-row)

- The "selected" state is propagated to the neighboring hits

- The previous hit is now readout 


\section{Grid Clustering - Select and Readout}

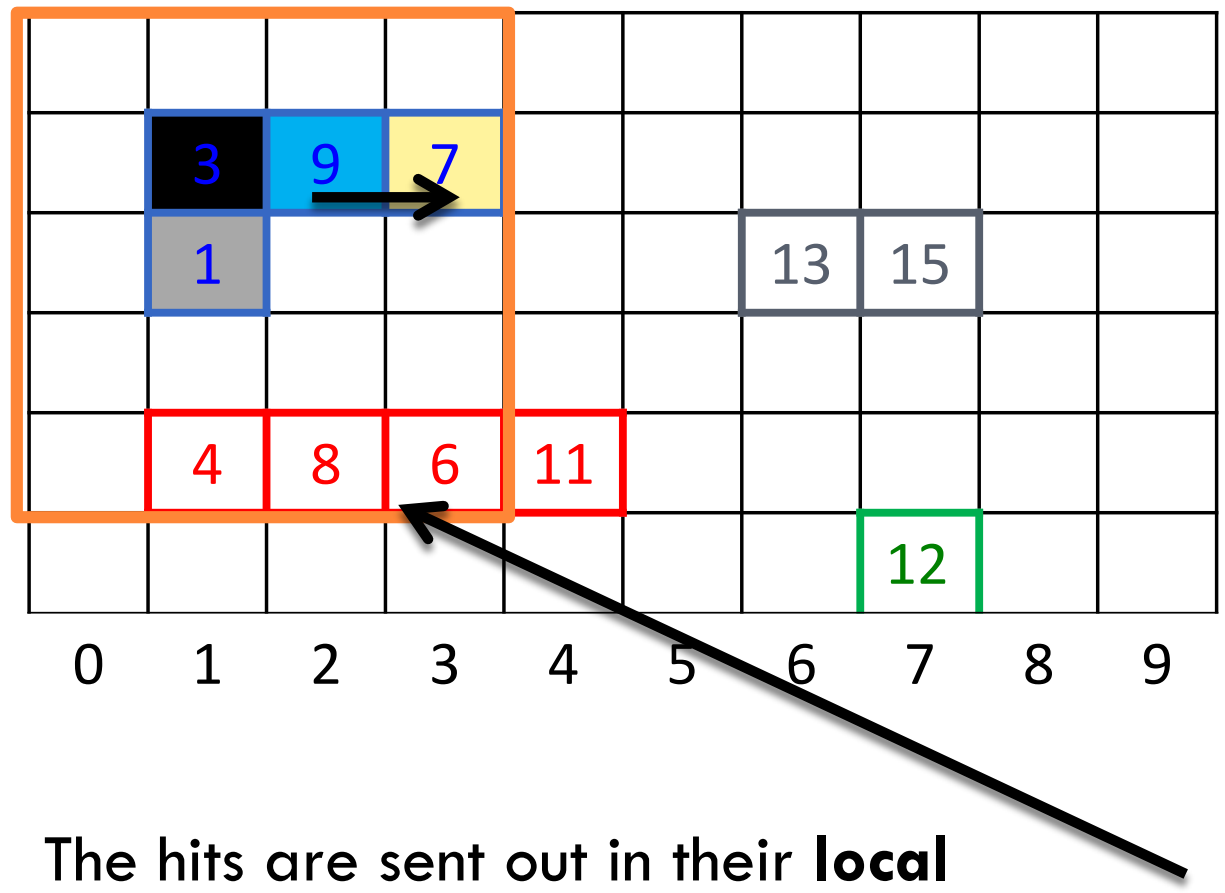

coordinates with regards to the reference hit

- Less bits for calculation in the next processing element

- The reference hit is sent out in absolute coordinates in the end cluster word
- Two cells in the cluster window are selected as "seeds" $(0$, mid-row) and (1, mid-row)

- The "selected" state is propagated to the neighboring hits

- The previous hit is now readout

- Selecting and reading out the cluster is executed in parallel

- The hits that do not belong to the cluster are recovered and written to the circular buffer in their absolute coordinates

- 8 bits of the end_event word are used for error flags 


\section{Grid Clustering - Block Diagram}

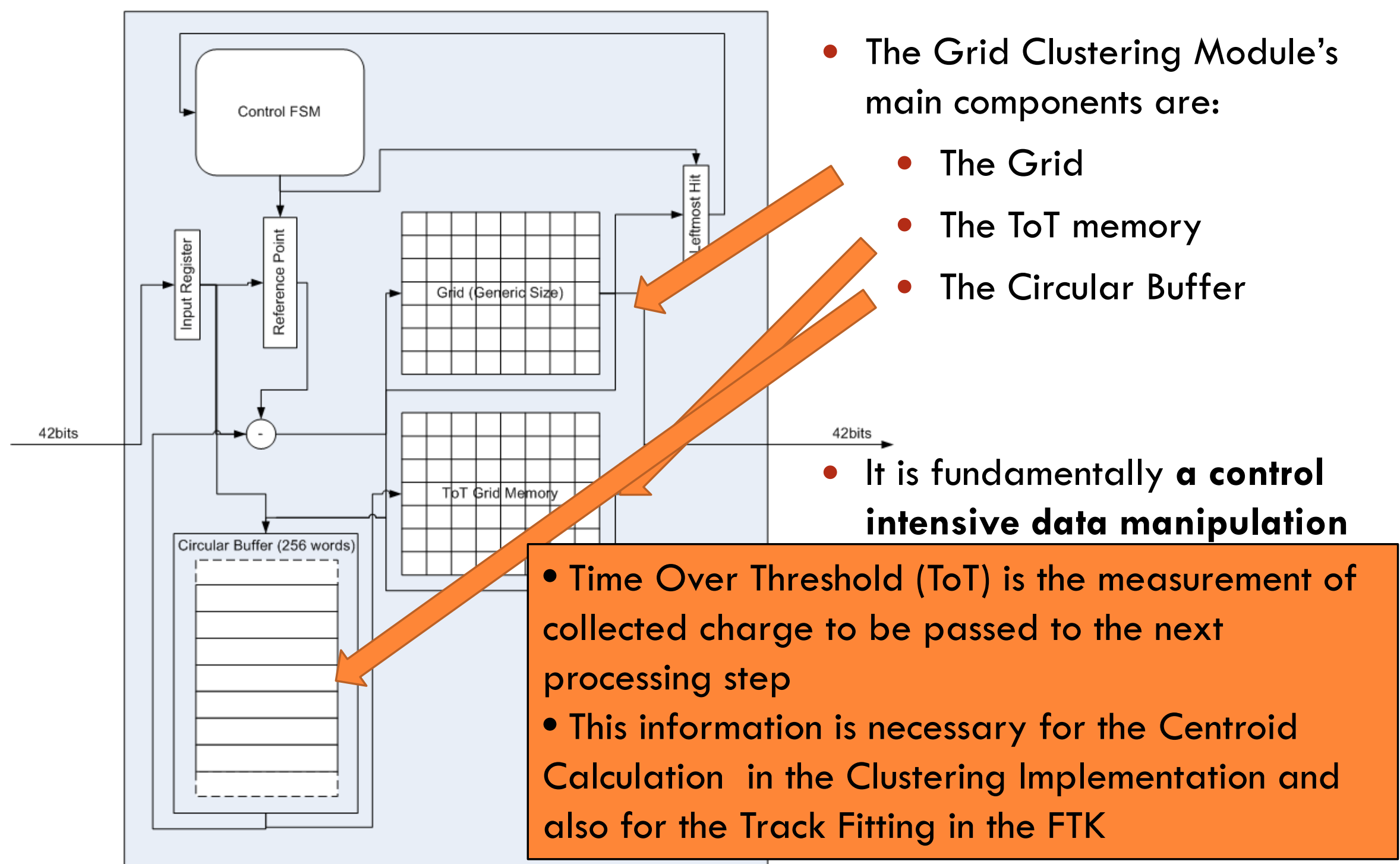




\section{Grid Clustering - The Grid}

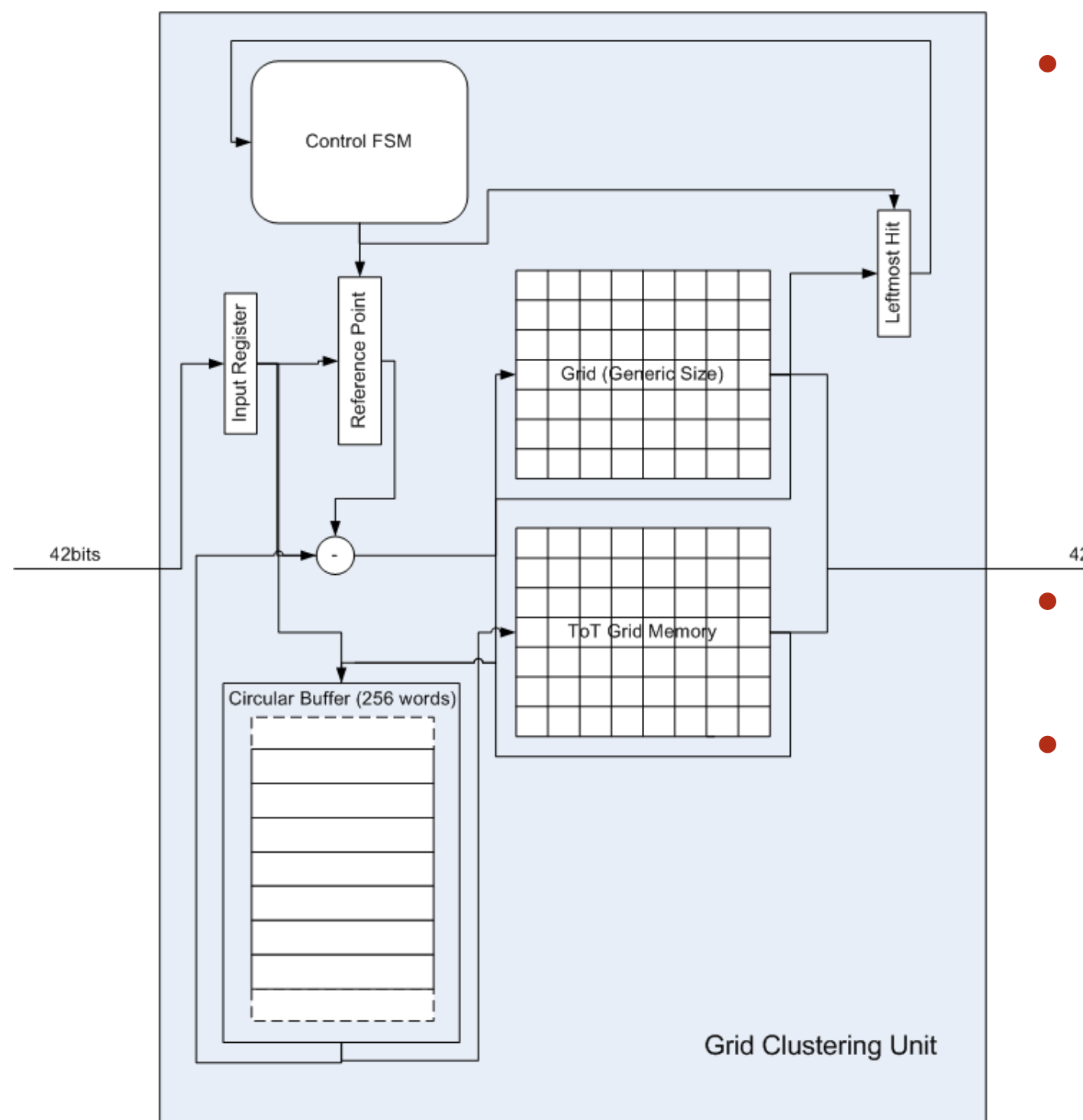

- The Grid is based on a preexisting algorithm, with some simplifications required because

- it now has a smaller size than in its initially proposed implementation

- a reference hit is defined

42bits,

- The current Grid size chosen is $21 \times 8$ pixels

- Pixels exceeding this size will be split at the boundary (and recovered in the post-processing step) 


\section{Grid Clustering - ToT Memory}

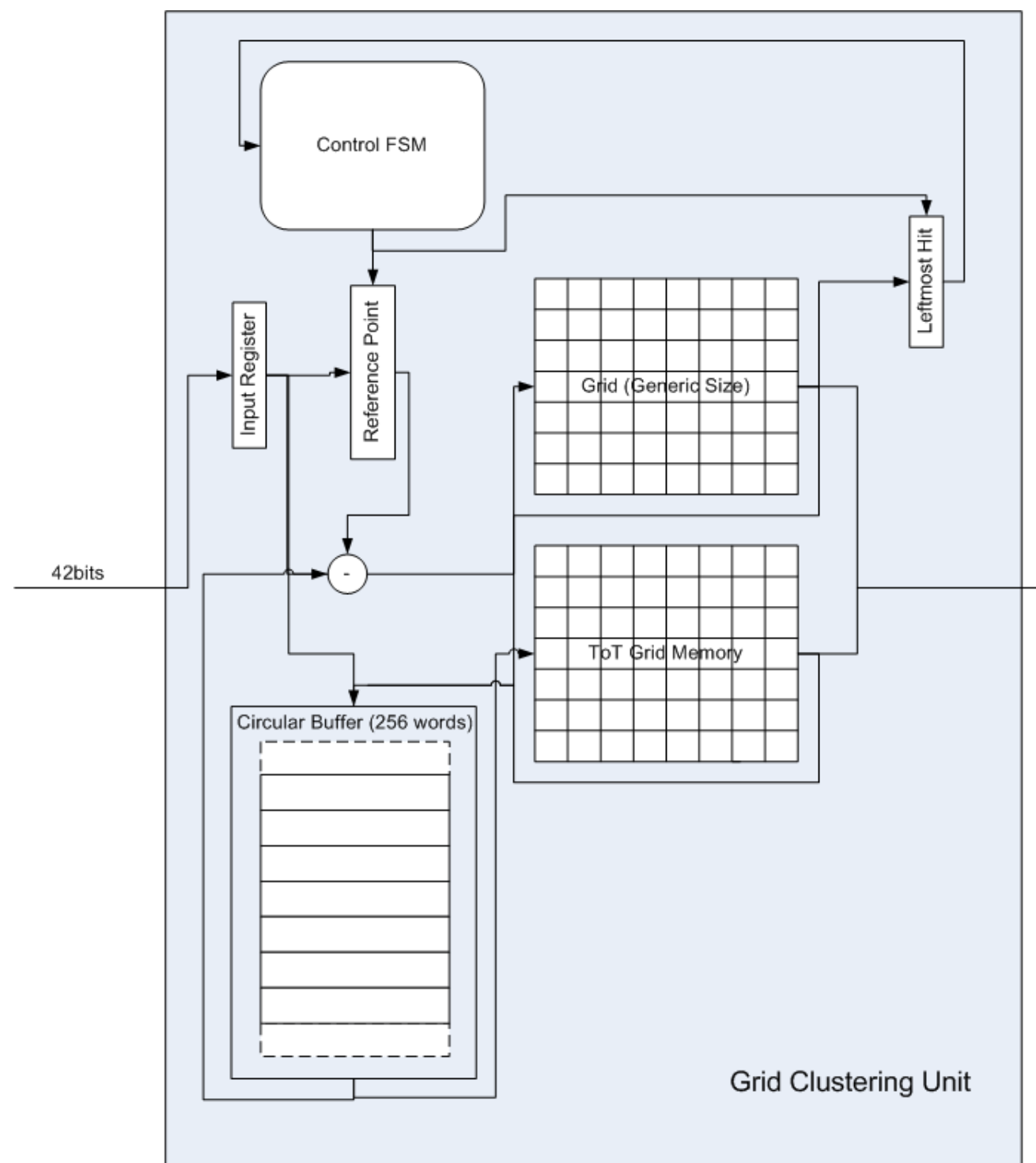

- The ToT Memory is a single port RAM of 256 words implemented on BRAM

(A BRAM is the configurable memory module on an FPGA device)

- In this memory the ToT (TimeOverThreshold) values of the hits loaded in the Grid are stored 42bits

- The stored values are addressed by their coordinates in a way that

- allows the memory size to be generic

- minimizes the imposed delay 


\section{Grid Clustering - Circular Buffer}

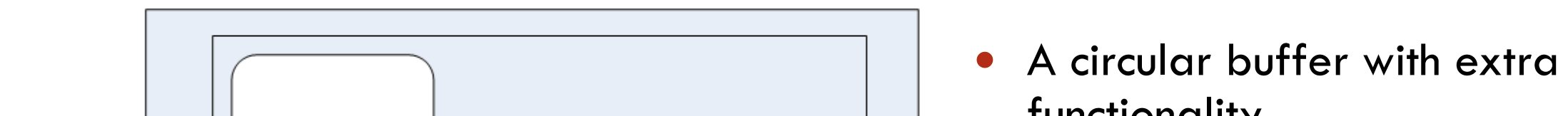

functionality

- Hits are read and written simultaneously, which can cause data to be read multiple times in the same operational state $\rightarrow$

$-\quad$ Control FSM

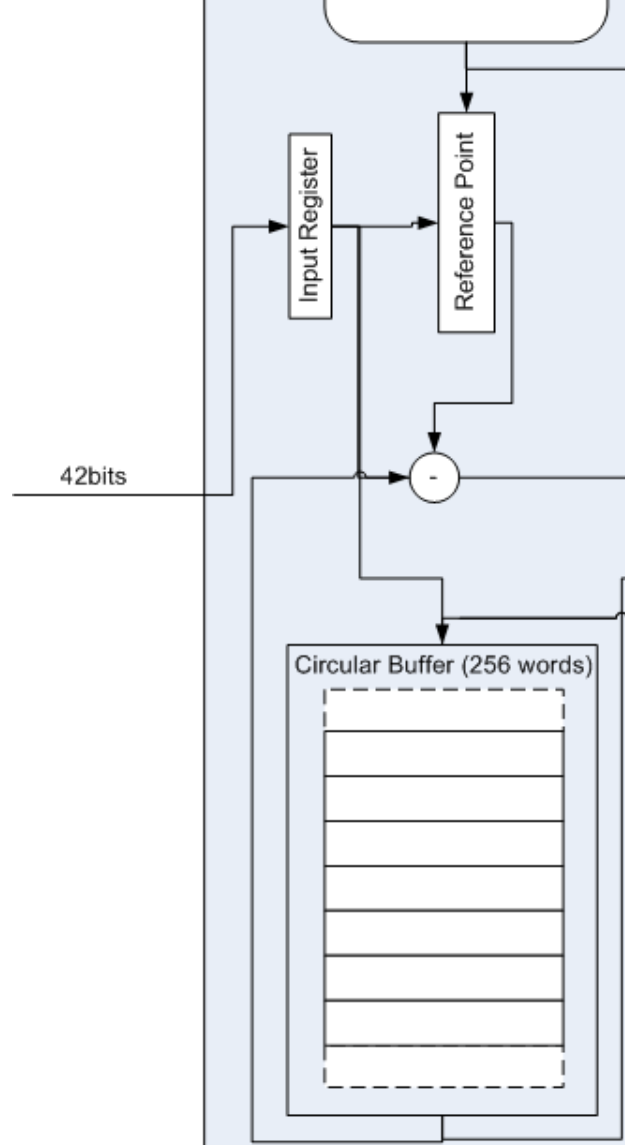

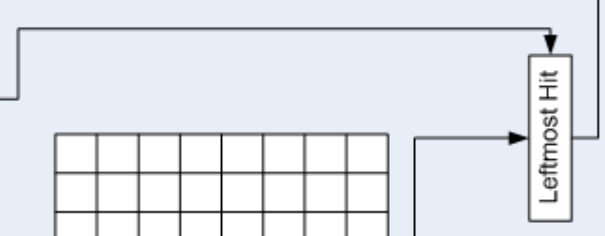

Grid (Gererio Size)

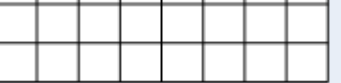

42 bits

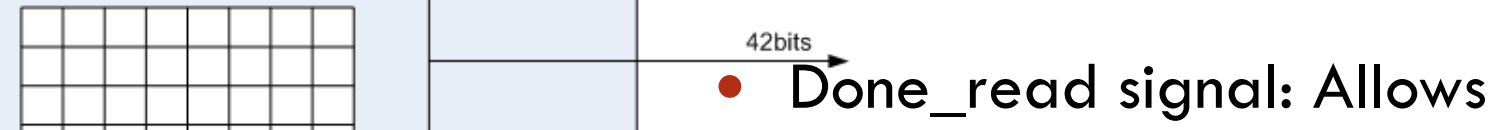
reading of hits up to a previously stored value of the write_pointer 


\section{Grid Clustering - Circular Buffer}

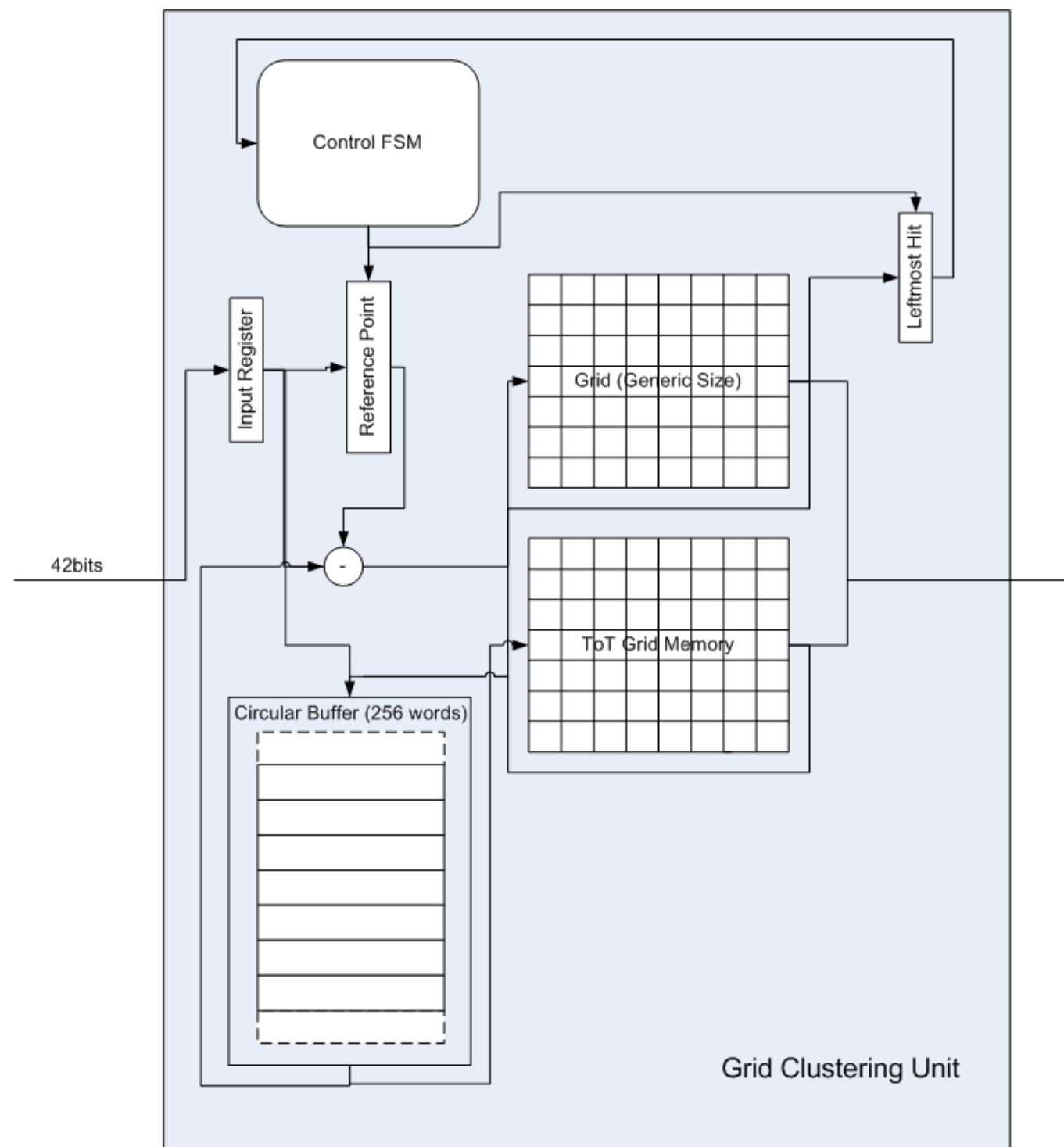

- Everytime a hit is written to the circular buffer it is compared to the value of the leftmost register

- The leftmost register stores the value of the hit in the circular buffer with the leftmost column value

42bits

- This hit will become the reference hit when data are recovered from the circular buffer to be loaded to the Grid

- The process continues until an end_event or a module_trailer arrives and the circ_buff is empty 


\section{Grid Clustering - Current State}

- The Grid Clustering module is fully functional

- Successfully passed post-place\&route simulation with a 12ns clock on a Spartan 6-LX150T FPGA

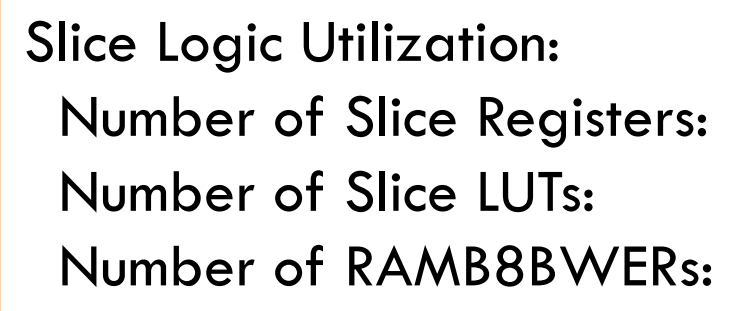

Number of Slice Registers:

Number of Slice LUTs:

Number of RAMB8BWERs:

$$
\begin{aligned}
& 816 \text { out of } 184,304 \quad 1 \% \\
& 2,021 \text { out of } 92,152 \quad 2 \% \\
& 2 \text { out of } \quad 536 \quad 1 \%
\end{aligned}
$$

- Typical algorithm execution will require on average $O(10)$ clock cycles for each input hit

- The number of clock cycles/hit will depend on module occupancy 


\section{Grid Clustering - Performance}

- The Grid Clustering module has achieved an operational frequency of $80 \mathrm{MHz}$

- For $80 \mathrm{MHz} / \sim 10$ clock $/$ hit $\rightarrow \sim 8 \mathrm{MHz}$ per hit

- By implementing 10 copies of the of the module: 10 parallel copies $\rightarrow \sim 80 \mathrm{MHz}$ hit processing rate $\rightarrow 20 \%$ FPGA device area occupied

- An increase even to a parallelism of 15 to 20 modules can be achieved with even better performance results 


\section{The Clustering Implementation}

- The current implementation is an evolution of a linear algorithm with a high cost in terms of FPGA resources

- In the previous algorithm grids of $168 \times 4$ or $328 \times 8$ pixels were used. For these grid sizes the extrapolated area and clock results (for the Spartan 6-LX 150T) would be:

\begin{tabular}{|l|r|r|r|r|}
\hline \multicolumn{1}{|c|}{ Grid Size } & Slice Registers & \multicolumn{1}{|c|}{ Slice LUTs } & Clock & Frequency \\
\hline $21 \times 8$ (current) & $696(1 \%)$ & $1950(2 \%)$ & $12 \mathrm{~ns}$ & $83 \mathrm{Mhz}$ \\
\hline $168 \times 4$ & $2784(1.5 \%)$ & $7800(8.2 \%)$ & $68 \mathrm{~ns}$ & $14.8 \mathrm{Mhz}$ \\
\hline $328 \times 8$ & $10510(5.7 \%)$ & $30457(33 \%)$ & $265 \mathrm{~ns}$ & $3.8 \mathrm{Mhz}$ \\
\hline
\end{tabular}

- In the new version of the algorithm the logic of each grid cell is much reduced 


\section{Clustering Modules}
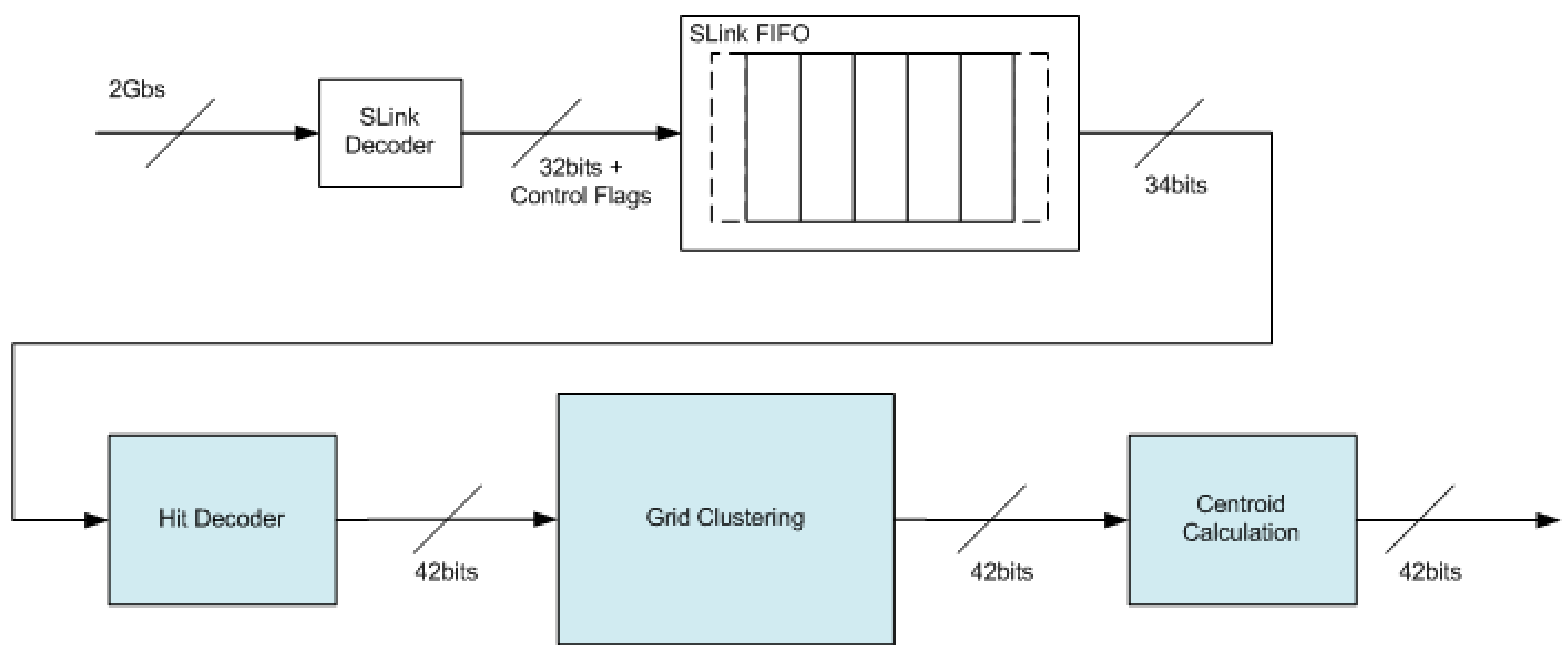


\section{Clustering Modules}
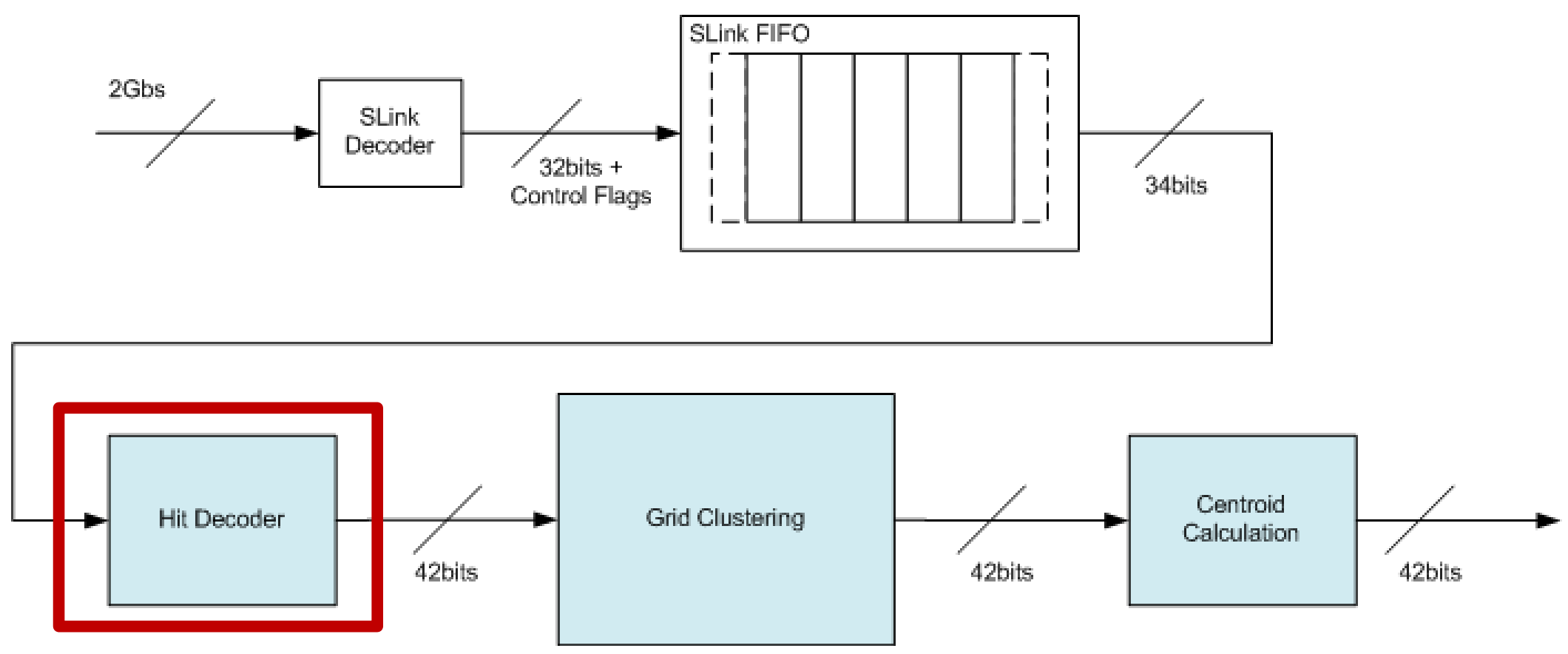


\section{Hit Decoder - Issues addressed}

ATLAS Pixel Bytestream Format

\begin{tabular}{|c|c|}
\hline Module header & OOIPAIbXMMMMMMMS5SSLLLEBEBEBEB \\
\hline [Mochle hit] & 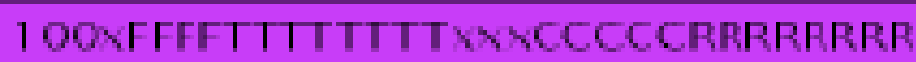 \\
\hline$[\ldots]$ & 100xFFFFTITTTTTXXCCCCCRRRRRRRR \\
\hline [Module entors] & \\
\hline Mochule Tailet & 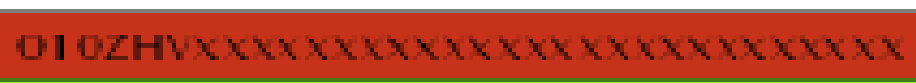 \\
\hline [...] & OOIPAILXXMMMMMMMLLLLLLL BBEBEBEBE \\
\hline$[\ldots]$ & 100XFFFTIITTTTTXXXCCCCRRRRRRRR \\
\hline$[\ldots]$ & \\
\hline 17 & \\
\hline
\end{tabular}

- Transform the input data to the format needed, dropping all unnecessary information (bits)

- Trace and fix errors of missing trailer words (end module, end event).

- Drop hits from unidentified events and modules 


\section{Hit Decoder - Issues addressed}

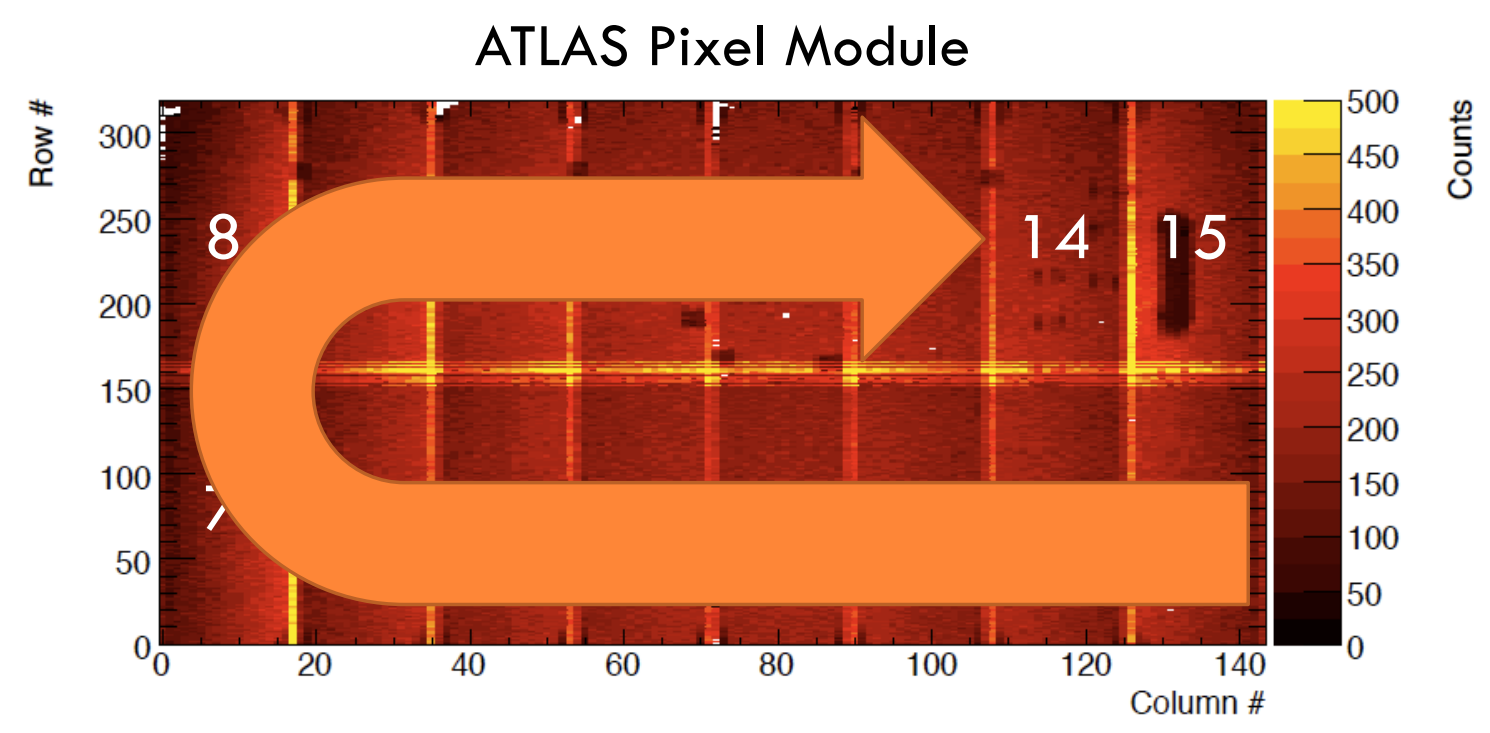

- FE chip numbering is circular

- FE chips are readout in the order of their numbering $\rightarrow$

- Incoming data is not in sequence:

- Hits from FE $<8$ come in the reverse column order

- Hits from FE $>=8$ come in the correct column order 


\section{Hit Decoder - Block Diagram}

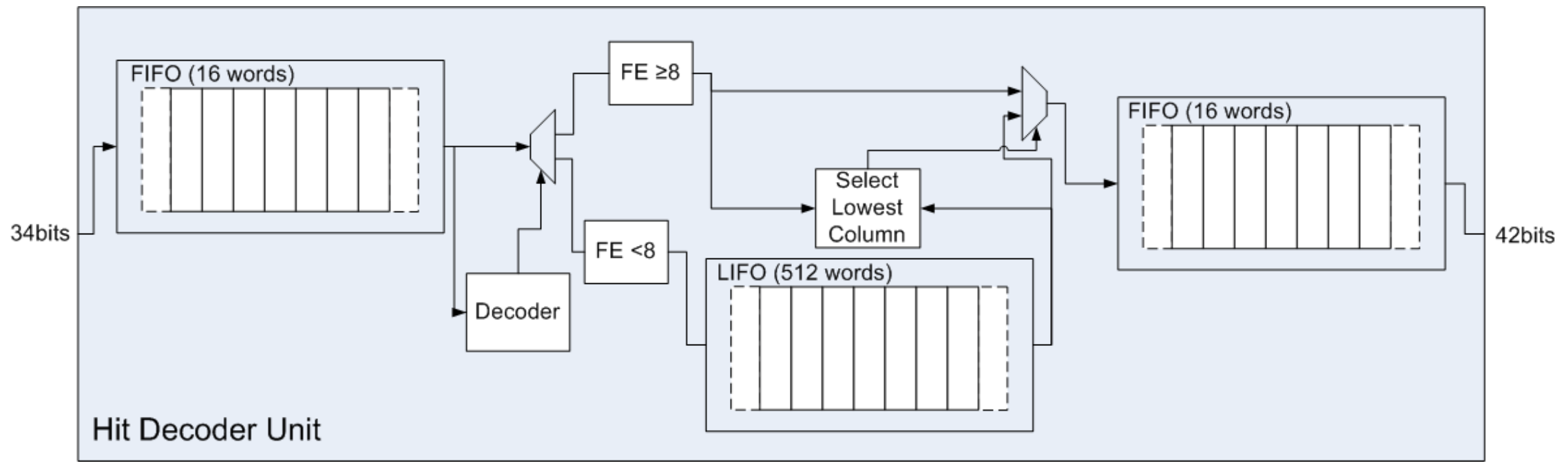

- Hits from $\mathrm{FE}<8$ are stored in a LIFO

- When a hit from FE $>=8$ arrives it is stored in a register

- The column value is compared with the last LIFO word

- The hit with the smallest column value is propagated to the output FIFO

- FIFOs are used to separate functions and simplify control logic

- Input and Output FIFOs could be used for possible Clock Domain crossing 


\section{Clustering Modules}
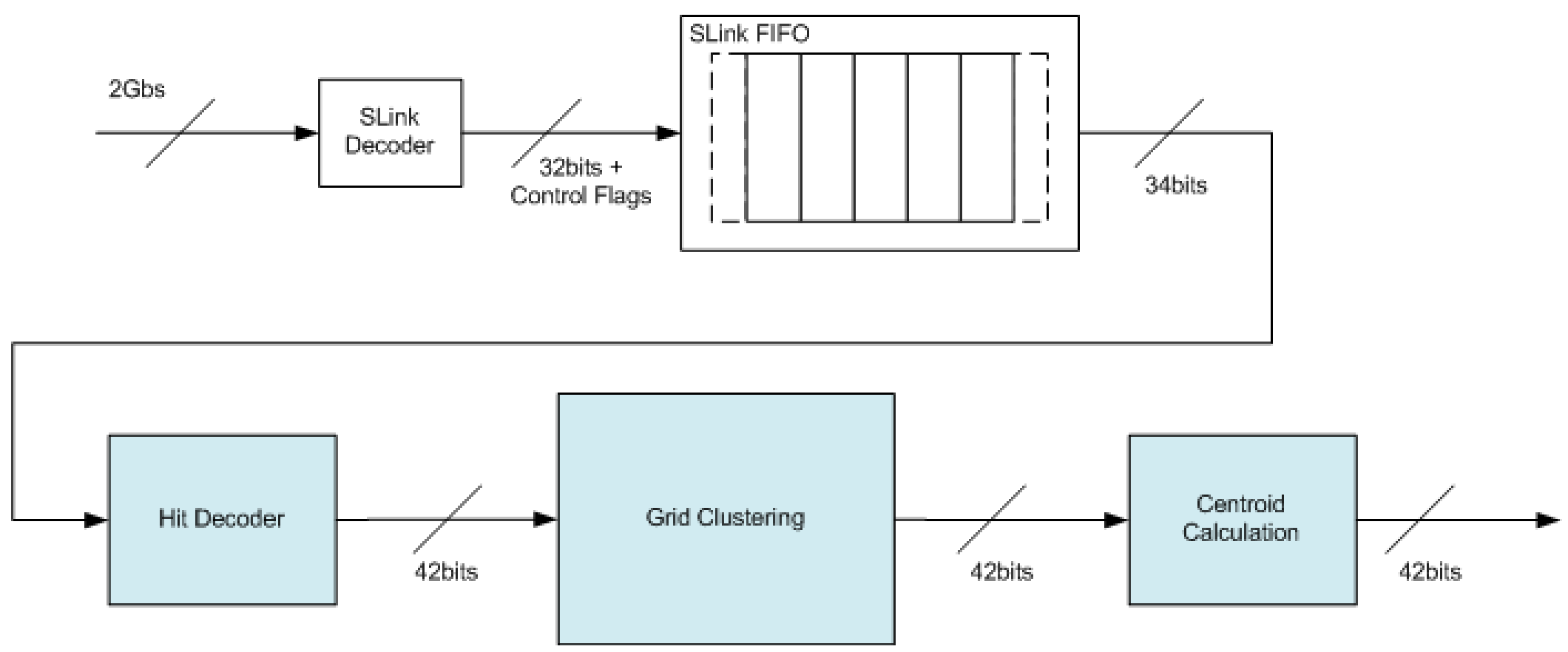


\section{Clustering Modules}
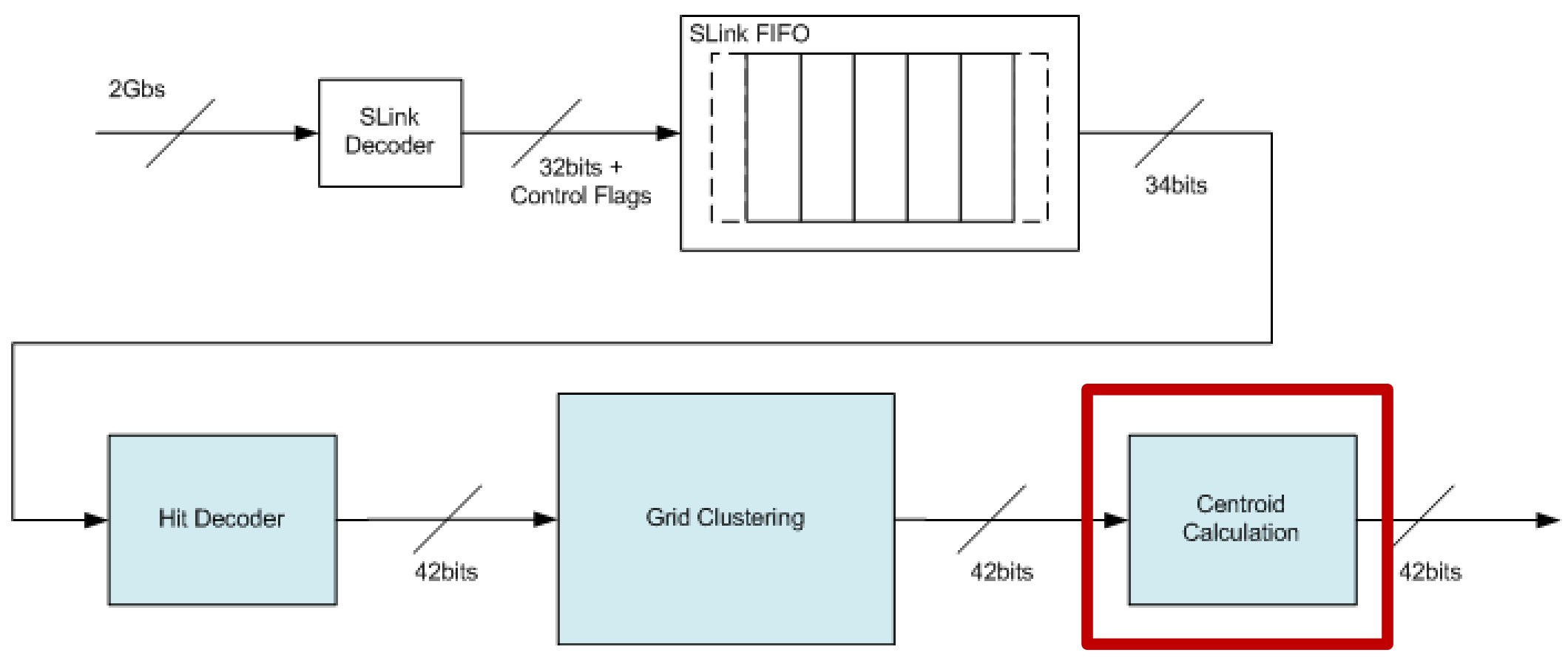


\section{Clustering Modules - Centroid Calculation}

- The post-processing step in the Clustering system is the one that actually performs the data reduction:

It reduces the set of cluster coordinates to one

- FTK cluster centroid is calculated as linear interpolation of deposited charge

- The Centroid Calculation step will output the cluster centroid and also the cluster size in two coordinates

- A center of mass can also be used (will not be used for FTK) for different applications (e.g. image processing) 


\section{Clustering Modules - Centroid Calculation}

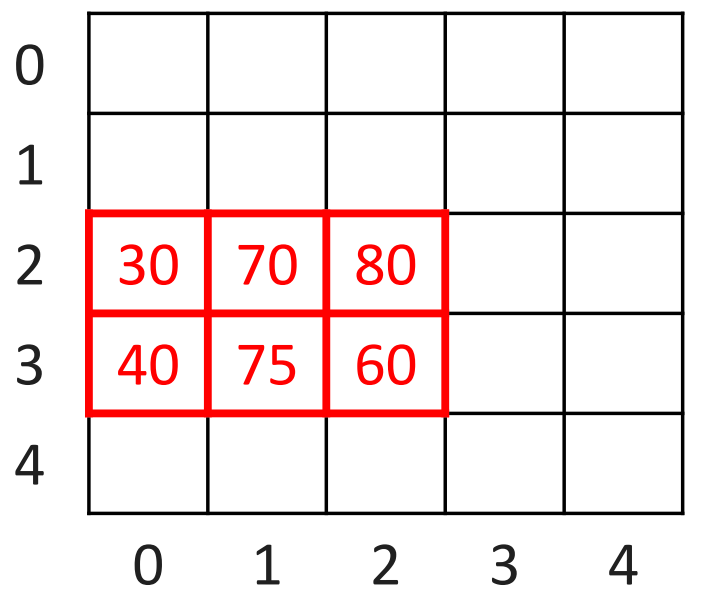

$$
\begin{gathered}
\text { qRatio }=\frac{q \text { ColMax }}{\text { qColMin }+ \text { qColMax }}=\frac{80+60}{(30+40)+(80+60)}=\frac{140}{210}=\frac{2}{3} \\
x_{-} \text {average }=\frac{\begin{array}{|c|c|c|}
\frac{\text { ColMin }+ \text { ColMax }}{2} \\
\text { Centroid }
\end{array} \text { Correction }}{\text { CRatio }-0.5)}
\end{gathered}
$$

- Constant $\boldsymbol{a}$ is a function of pixel position

- Calculate the charging balance between the two sides of the cluster (left-right for $y$, top-bottom for $x$ )

- Apply a correction with respect to the centroid

- For the above $3 \times 2$ cluster the centroid coordinates would be $(1.15,2.5)$ 


\section{Clustering Modules - Parallelization}

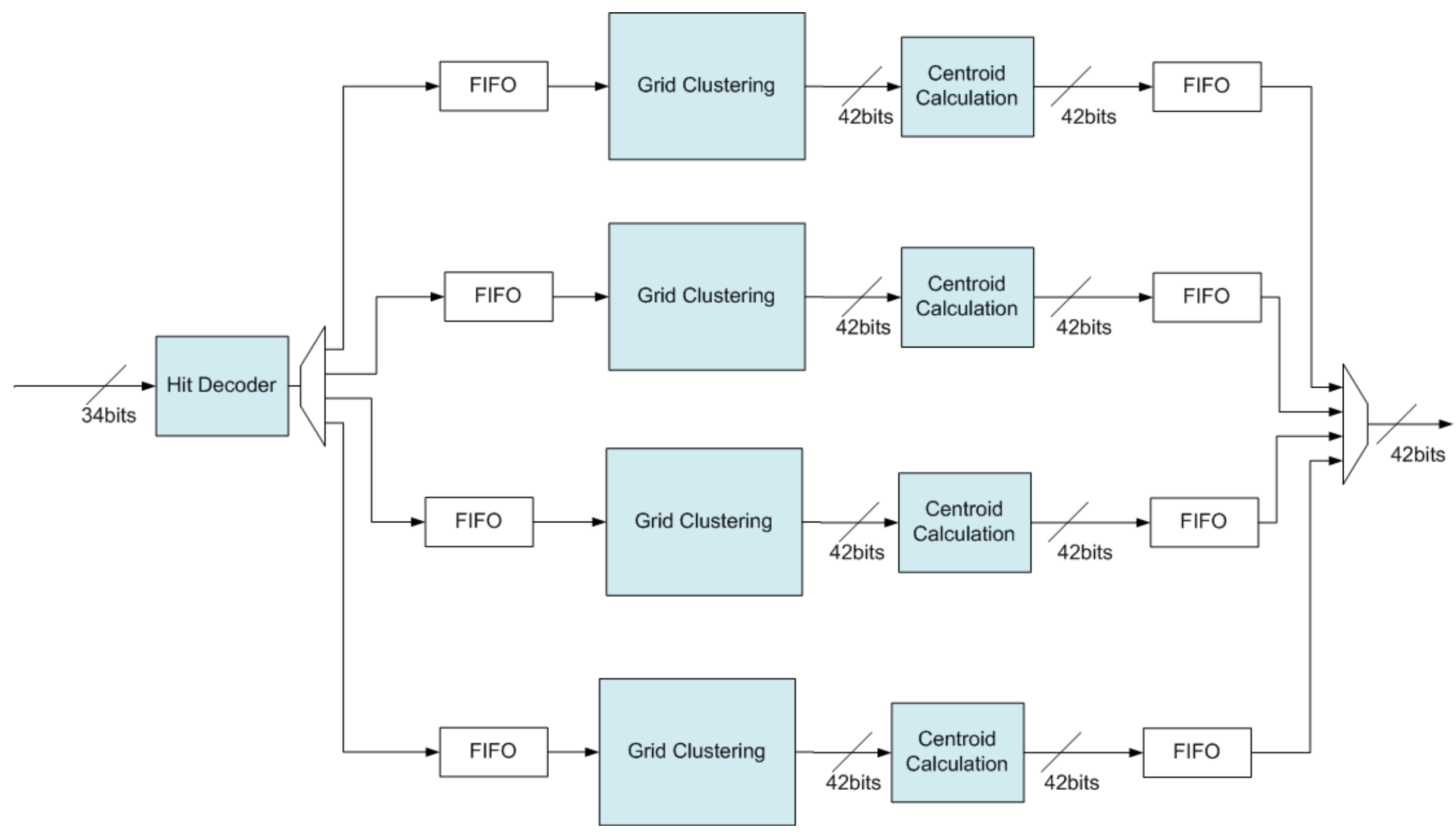




\section{Clustering Modules - Parallelization}

- The Grid Clustering and Center of Mass modules are working independently in different streams of data

- Each stream consists of data from the same module

- The data is tagged by its event and module number

- In the read out process data must be read in the proper event sequence

- The module number sequence in the read out process is irrelevant since the system will propagate each module data to a separate AM chip

- The full algorithm keeps up with the $40 \mathrm{MHz}$ input rate 


\section{Conclusions - Summary}

- A 2D-Clustering implementation was presented

- It targets the ATLAS FastTracKer but it is generic enough to be used in general image processing applications

- The module achieves a $\sim 80 \mathrm{MHz}$ operational frequency with 1\% FPGA slice Registers and 2\% slice LUTs occupation

- An estimated $\sim 10$ cycles per hit are necessary for cluster identification (The numbers will be verified by FTK simulation in the near future)

- With $\times 10$ parallelization a $\sim 80 \mathrm{MHz}$ input rate will be covered (the specifications call for $40 \mathrm{MHz}$ ) 


\section{Acknowledgements}

- The presented work was supported by Istituto Nazionale di Fisica Nucleare, and European community FP7 funds (Marie Curie IAPP Project 324318 FTK).

Thank you!
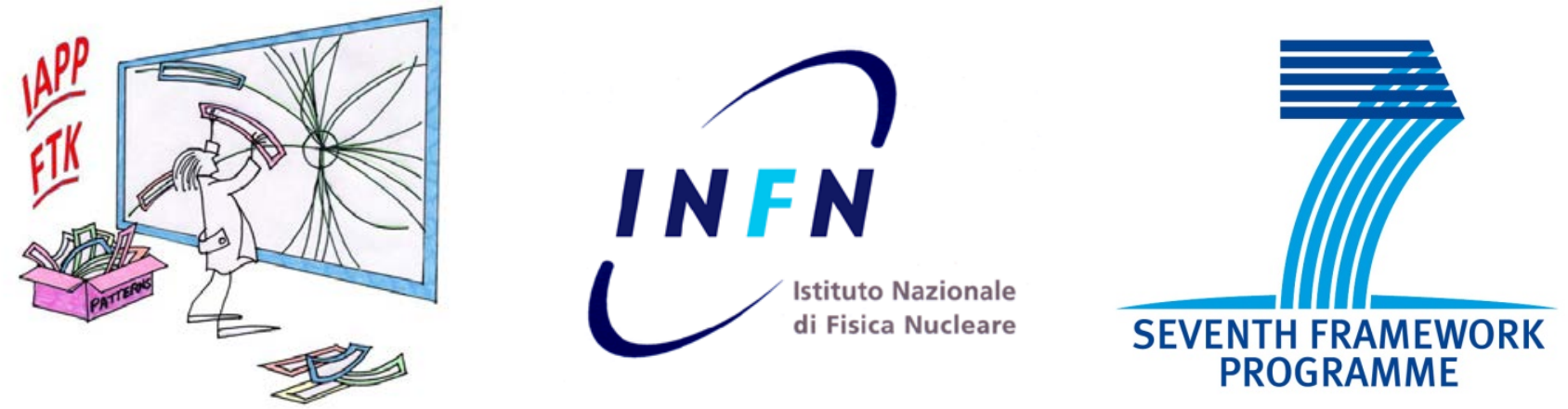


\section{BackUp Slides - Data Format}

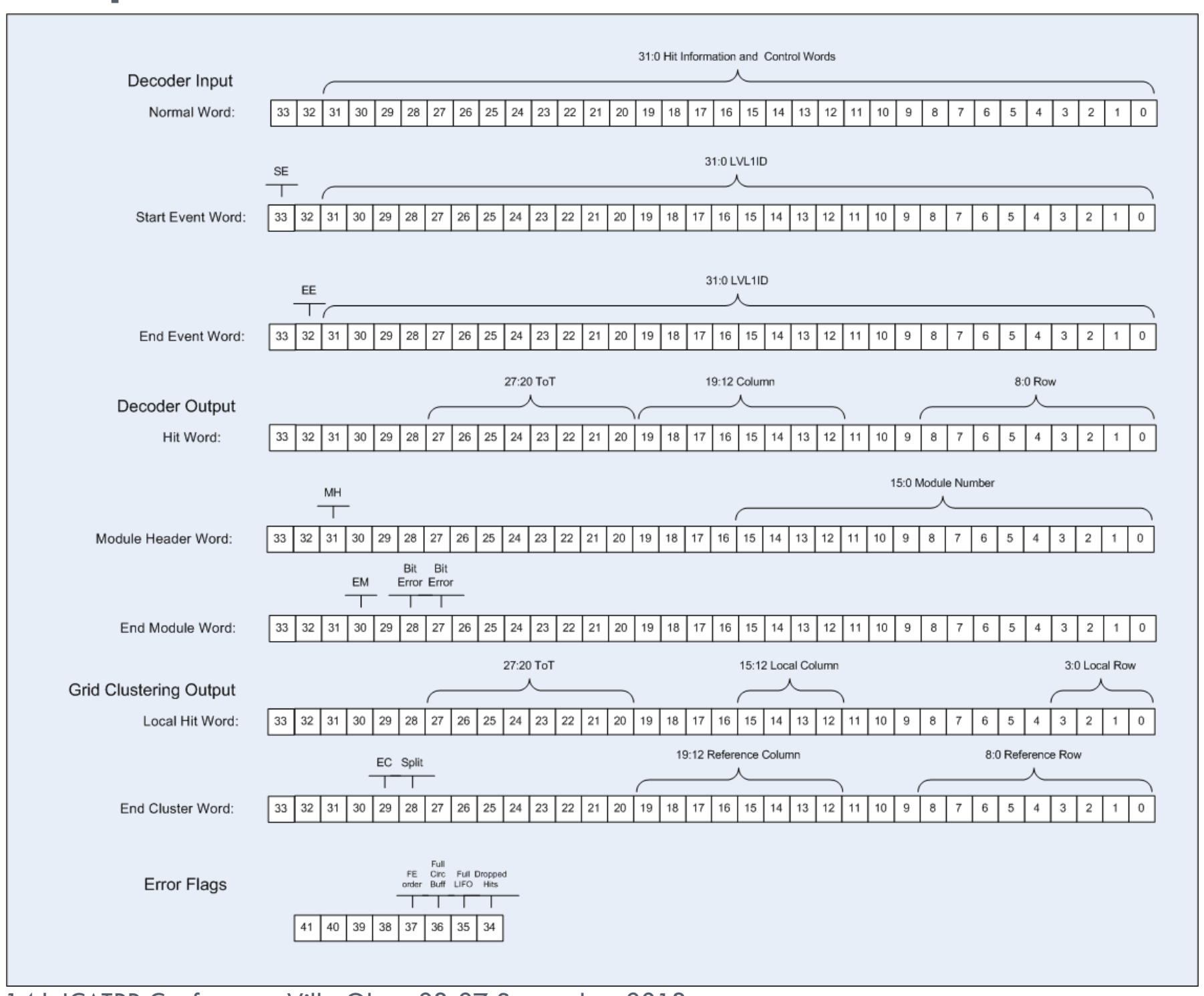

C.- L. Sotiropoulou - 14th ICATPP Conference, Villa Olmo, 23-27 September 2013 


\section{BackUp Slides - Centroid Calculation}

\section{Calculation process}

- Find the smallest and largest pixel index for column or row

- Sum the ToT (deposited charge estimator) for all pixels in the smallest and largest column or row

- Center is calculated as:

- qRatio_y = qRowMax/float(qRowMin+qRowMax);

- qRatio_x=qColMax/float(qColMin+qColMax);

- deltax $=30 *$ micrometer* (sensorThickness/(250* micrometer));

- deltay = sensorThickness*fabs(pixelEstimateCotTheta);

- $y_{\text {_average }}=($ rowMin+rowMax $) / 2 .+\operatorname{deltax} *($ etaRow-0.5 $) /$ phiPitch;

- $x_{\text {_average }}=($ colMin+colMax $) / 2 .+$ deltay $*($ etaCol-0.5 $) /$ etaPitch;

- where pixelEstimateCotTheta is an estimate of the CotTheta obtained from (colMin+colMax)/2. 